\title{
Flexible High-Output Nanogenerator Based on Lateral ZnO Nanowire Array
}

\author{
Guang Zhu, ${ }^{\dagger}$ Rusen Yang, ${ }^{\dagger}$ Sihong Wang, and Zhong Lin Wang* \\ School of Materials Science and Engineering, Georgia Institute of Technology, Atlanta, Georgia 30332-0245
}

\begin{abstract}
We report here a simple and effective approach, named scalable sweeping-printing-method, for fabricating flexible highoutput nanogenerator (HONG) that can effectively harvesting mechanical energy for driving a small commercial electronic component. The technique consists of two main steps. In the first step, the vertically aligned ZnO nanowires (NWs) are transferred to a receiving substrate to form horizontally aligned arrays. Then, parallel stripe type of electrodes are deposited to connect all of the NWs together. Using a single layer of HONG structure, an open-circuit voltage of up to $2.03 \mathrm{~V}$ and a peak output power density of $\sim 11 \mathrm{~mW} / \mathrm{cm}^{3}$ have been achieved. The generated electric energy was effectively stored by utilizing capacitors, and it was successfully used to light up a commercial light-emitting diode (LED), which is a landmark progress toward building self-powered devices by harvesting energy from the environment. This research opens up the path for practical applications of nanowire-based piezoelectric nanogeneragtors for self-powered nanosystems
\end{abstract}

KEYWORDS Nanogenerator, ZnO, nanowire, light-emitting diode, self-powering

$\mathrm{E}$ nergy harvesting is critical to achieve independent and sustainable operations of nanodevices, aiming at building self-powered nanosystems. ${ }^{1-3}$ Taking the forms of irregular air flow/vibration, ultrasonic waves, body movement, and hydraulic pressure, mechanical energy is ubiquitously available in our living environment. It covers a wide range of magnitude and frequency from cell contraction to ocean waves. The mechanical-electric energy conversion has been demonstrated using piezoelectric cantilever working at its resonating mode. ${ }^{4-7}$ However, the applicability and adaptability of the traditional cantilever based energy harvester is greatly impeded by the large unit size, large triggering force and specific high resonance frequency. Recently, a series of rationally designed nanogenerators (NGs) with piezoelectric nanowires (NWs) have shown great potential to scavenge tiny and irregular mechanical energy. ${ }^{8-15}$ However, insufficient electric output hinders their practical applications. We report here a simple and effective approach, named scalable sweeping-printing-method, for fabricating flexible high-output nanogenerator (HONG). An open-circuit voltage of up to $2.03 \mathrm{~V}$ and a peak output power density of $\sim 11 \mathrm{~mW} / \mathrm{cm}^{3}$ have been achieved. The generated electric energy was effectively stored by utilizing capacitors, and it was successfully used to light up a commercial lightemitting diode (LED), which is a landmark progress toward building self-powered devices by harvesting energy from the environment. Furthermore, by optimizing the density of the NWs on the substrate and with the use of multilayer integration, a peak output power density of $\sim 0.44 \mathrm{~mW} / \mathrm{cm}^{2}$ and volume density of $1.1 \mathrm{~W} / \mathrm{cm}^{3}$ are predicted.

*To whom correspondence should be addressed. E-mail: zlwang@gatech.edu.

† Authors with equal contribution

Received for review: $6 / 3 / 2010$

Published on Web: 07/21/2010
The mechanism of converting mechanical energy by a single $\mathrm{ZnO} N \mathrm{NW}$ that is laterally bonded to a substrate has been discussed in details in our previous report. ${ }^{13}$ Owing to much smaller diameter of the NW compared to the substrate thickness, outward bending of the substrate induces a uniaxial tensile strain in the NW. Because of the piezoelectric property of the $\mathrm{ZnO} \mathrm{NW}$, the stress results in a piezoelectric field along the length, which causes a transient charge flow in the external circuit. The Schottky contact at the bonded ends can regulate the charge flow. As a result, the bending and releasing of the single-wire-NG gives rise to an alternating flow of the charges in the external circuit. In this work, the power output has been scaled up with the integration of hundreds of thousands of horizontally aligned NWs, which was made by a scalable sweeping-printing-method that is simple, cost-effective, and highly efficient.

The method consists of two main steps. In the first step, the vertically aligned NWs are transferred to a receiving substrate to form horizontally aligned arrays. The major components of the transfer setup are two stages (Figure 1a). Stage 1 has a flat surface that faces downward and holds the vertically aligned NWs; stage 2 has a curved surface and holds the receiving substrate. Polydimethylsiloxane (PDMS) film on the surface of stage 2 is used as a cushion layer to support the receiving substrate and enhances the alignment of the transferred NWs. The radius of the curved surface of stage 2 equals the length of the rod supporting the stage, which is free to move in circular motion (Supporting Information Figure S1). In the second step, electrodes are deposited to connect all of the NWs together.

Vertically aligned ZnO NWs on Si substrates were synthesized using physical vapor deposition method. ${ }^{16,17}$ The dense and uniform NWs have the length of $\sim 50 \mu \mathrm{m}$, diameter of $\sim 200 \mathrm{~nm}$, and growth direction along the $c$-axis 


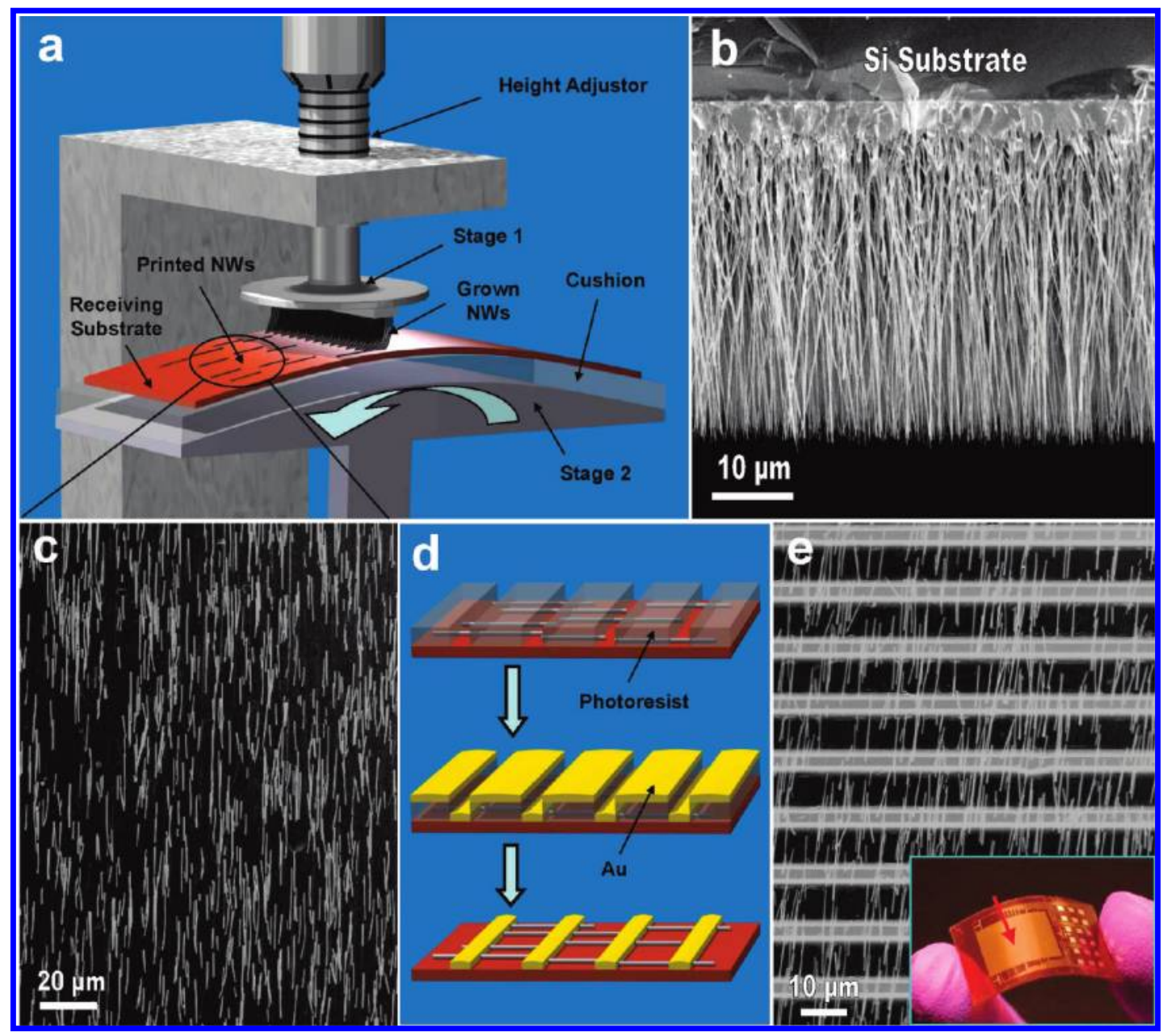

FIGURE 1. Fabrication process and structure characterization of the HONG. (a) Experimental setup for transferring vertically grown ZnO NWs to a flexible substrate to make horizontally aligned ZnO NW arrays with crystallographic alignment. (b) SEM image of as-grown vertically aligned ZnO NWs by physical vapor method on Si substrate. (c) SEM image of the as-transferred horizontal ZnO NWs on a flexible substrate. (d) Process of fabricating Au electrodes on horizontal ZnO NW arrays, which includes photolithography, metallization, and lift-off. (e) SEM image of $\mathrm{ZnO} \mathrm{NW}$ arrays bonded by Au electrodes. Inset: demonstration of an as-fabricated HONG. The arrowhead indicates the effective working area of the HONG.

(Figure 1b, Supporting Information Figure S2). The same growth direction of NWs guarantees the alignment of the piezoelectric potentials in all of the NWs and a successful scaling up of the output, which will be elaborated later. A small piece of $\mathrm{Si}$ substrate with grown ZnO NWs was mounted onto stage 1 (Figure 1a) and a piece of Kapton film with the thickness of $125 \mu \mathrm{m}$ was attached to stage 2 (Figure 1a). The distance between the receiving substrate and NWs was precisely controlled to form a loose contact between the two. The receiving substrate then counterclockwise swept across the vertical NWs arrays, which were detached from Si substrate and aligned on the receiving substrate along the direction of sweeping due to the applied shear force (Figure 1a). The as-transferred NWs are presented in Figure $2 \mathrm{c}$ with an estimated average density of $1.1 \times 10^{6} \mathrm{~cm}^{-2}$. The length variation is probably due to the fact that not all of the NWs were broken off at the roots.

Next, the evenly spaced electrode pattern over the aligned NWs was first defined using photolithography and then followed by sputtering $300 \mathrm{~nm}$ thick Au film (Figure 1d). After lifting off the photoresist, 600 rows of stripe-shaped Au electrodes with $10 \mu \mathrm{m}$ spacing were fabricated on top of the horizontal NW arrays (Figure 1e). Au electrodes form Schottky contacts with the ZnO NWs, which are mandatory for a working NG. ${ }^{8,18}$ Approximately $3.0 \times 10^{5} \mathrm{NWs}$ in an effective working area of $1 \mathrm{~cm}^{2}$, as pointed by an arrowhead in Figure $1 \mathrm{~d}$ (inset), are in contact with electrodes at both ends. Finally, a PDMS packaging over the entire structure can further enhance mechanical robustness and protect the device from invasive chemicals.

The working principle of the HONG is illustrated by the schematic diagrams in Figure 2a,b. NWs connected in parallel collectively contribute to the current output; NWs in different rows connected in serial constructively improve the voltage output. The same growth direction of all NWs and the sweeping printing method ensure that the crystallographic orientations of the horizontal NWs are aligned along the sweeping direction. Consequently, the polarity of 


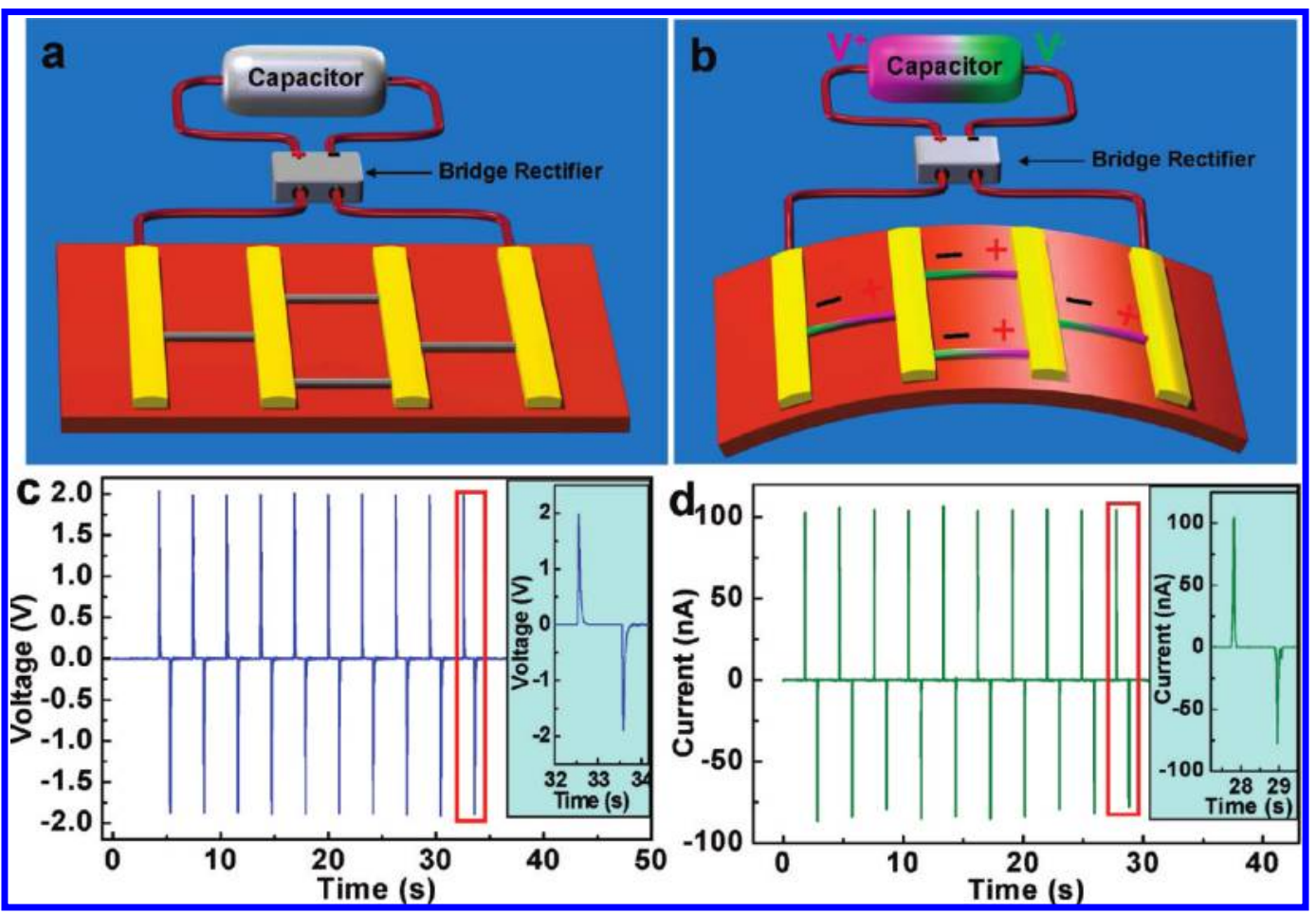

FIGURE 2. Working principle and output measurement of the HONG. (a) Schematic diagram of HONG's structure without mechanical deformation, in which gold is used to form Schottky contacts with the ZnO NW arrays. (b) Demonstration of the output scaling-up when mechanical deformation is induced, where the " \pm " signs indicate the polarity of the local piezoelectric potential created in the NWs. (c) Open circuit voltage measurement of the HONG. (d) Short circuit current measurement of the HONG. The measurement is performed at a strain of $0.1 \%$ and strain rate of $5 \% \mathrm{~s}^{-1}$ with the deformation frequency of $0.33 \mathrm{~Hz}$. The insets are the enlarged view of the boxed area for one cycle of deformation.

the induced piezopotential is also aligned, leading to a macroscopic potential contributed constructively by all of the NWs (Figure 2b).

To investigate the performance of the HONG, a linear motor was used to periodically deform the HONG in a cyclic stretching-releasing agitation $(0.33 \mathrm{~Hz})$. The open-circuit voltage $\left(V_{o c}\right)$ and the short-circuit current $\left(I_{\mathrm{sc}}\right)$ were measured with caution to rule out possible artifacts. ${ }^{19}$ At a strain of $0.1 \%$ and strain rate of $5 \% \mathrm{~s}^{-1}$, peak voltage and current reached up to $2.03 \mathrm{~V}$ and $107 \mathrm{nA}$, respectively. Assuming that all of the integrated NWs actively contribute to the output, the current generated by a single NW is averaged to be $\sim 200 \mathrm{pA}$; and the voltage from each row is $\sim 3.3 \mathrm{mV}$ in average. Considering the size of the working area of the nanogenerator $\left(1 \mathrm{~cm}^{2}\right)$ (Figure $1 \mathrm{e}$, inset), a peak output power density of $\sim 0.22 \mu \mathrm{W} / \mathrm{cm}^{2}$ has been achieved, which is over 20 -fold increase compared to our latest report based on a more complex design. ${ }^{14}$ For nanowires with the diameter of $\sim 200 \mathrm{~nm}$, the power volume density is $\sim 11$ $\mathrm{mW} / \mathrm{cm}^{3}$, which is $12-22$ times of that from PZT based cantilever energy harvester. ${ }^{6,7}$ The durability test and further characterization were performed, which prove the stability and robustness of the HONGs (Supporting Information Figure S3). Voltage linear superposition test verified the proposed working principle of the HONGs (Supporting Information Figure S4).
Further scaling up the power output is expected to be technically feasible. If NWs can be uniformly and densely packed as a monolayer over the entire working area, and all can actively contribute to the output, the maximum power area density is expected to reach $\sim 22 \mu \mathrm{W} / \mathrm{cm}^{2}$. The power volume density is anticipated to be improved up to $\sim 1.1 \mathrm{~W} / \mathrm{cm}^{3}$. With 20 layers of such NW arrays stacked together, the power area density would be boosted up to $\sim 0.44 \mathrm{~mW} / \mathrm{cm}^{2}$.

The performance of the HONG is affected by strain and strain rate. For a given strain rate $\left(5 \% \mathrm{~s}^{-1}\right)$, an increase in strain leads to a larger output (Figure 3a,b). Likewise, at a constant strain $(0.1 \%)$, the output is proportional to the strain rate (Figure $3 \mathrm{c}, \mathrm{d}$ ). Beyond a certain strain and strain rate, saturation of the magnitude occurs, probably due to the converse piezoelectric effect, which is the strain created by the piezopotential and it is opposite to the externally induced strain. It is noticed that $0.1 \%$ strain is sufficient to induce effective output, which is much smaller than the $6 \%$ fracture strain of the $\mathrm{ZnO} \mathrm{NW}$ predicted theoretically. ${ }^{20}$

Storing the generated energy and driving functional devices are extremely important steps toward practical applications of the nanogenerator. In this work, they were accomplished by using a charging-discharging circuit with two consecutive steps (Figure 4). The circuit function is determined by the status of a switch (Figure $4 \mathrm{a}$ inset). The 


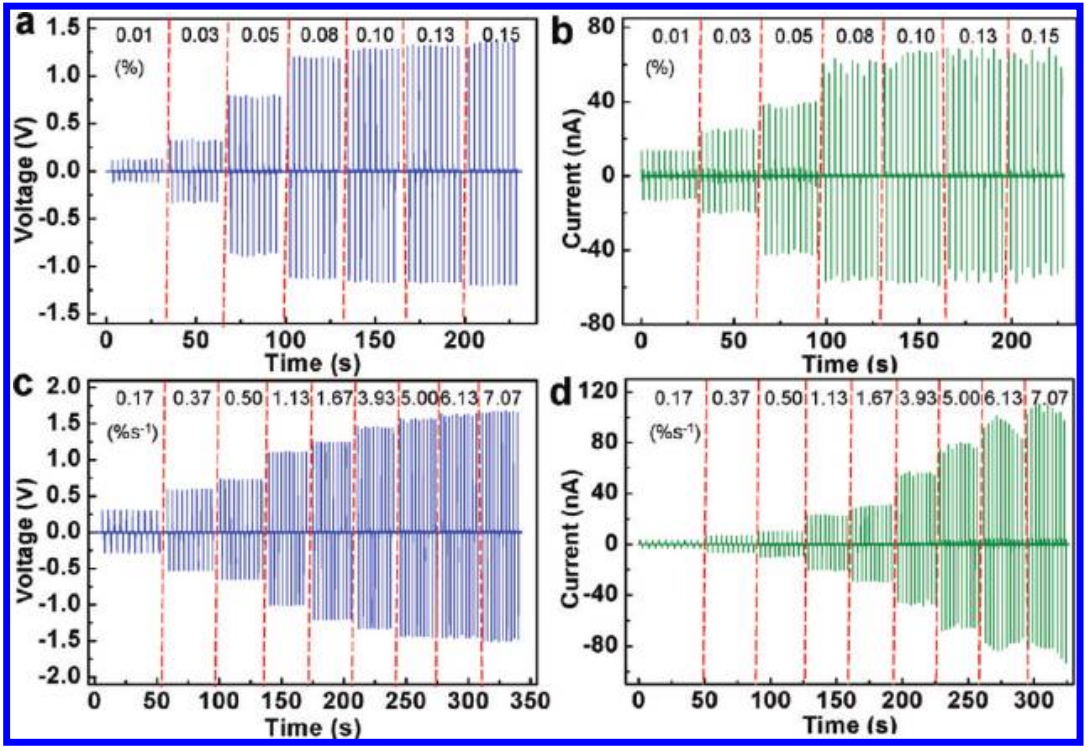

FIGURE 3. Performance characterization of the HONG with increasing strain and strain rate. (a) Open circuit voltage measurement of the HONG with increasing strain at a given strain rate of $5 \% \mathrm{~s}^{-1}$. (b) Short circuit current measurement of the HONG with increasing strain at a given strain rate of $5 \% \mathrm{~s}^{-1}$. (c) Open circuit voltage measurement of the HONG with increasing strain rate at a constant strain of $0.1 \%$. (d) Short circuit current measurement of the HONG with increasing strain rate at a constant strain of $0.1 \%$. For all measurements, the mechanical deformation frequency is fixed at $0.33 \mathrm{~Hz}$.

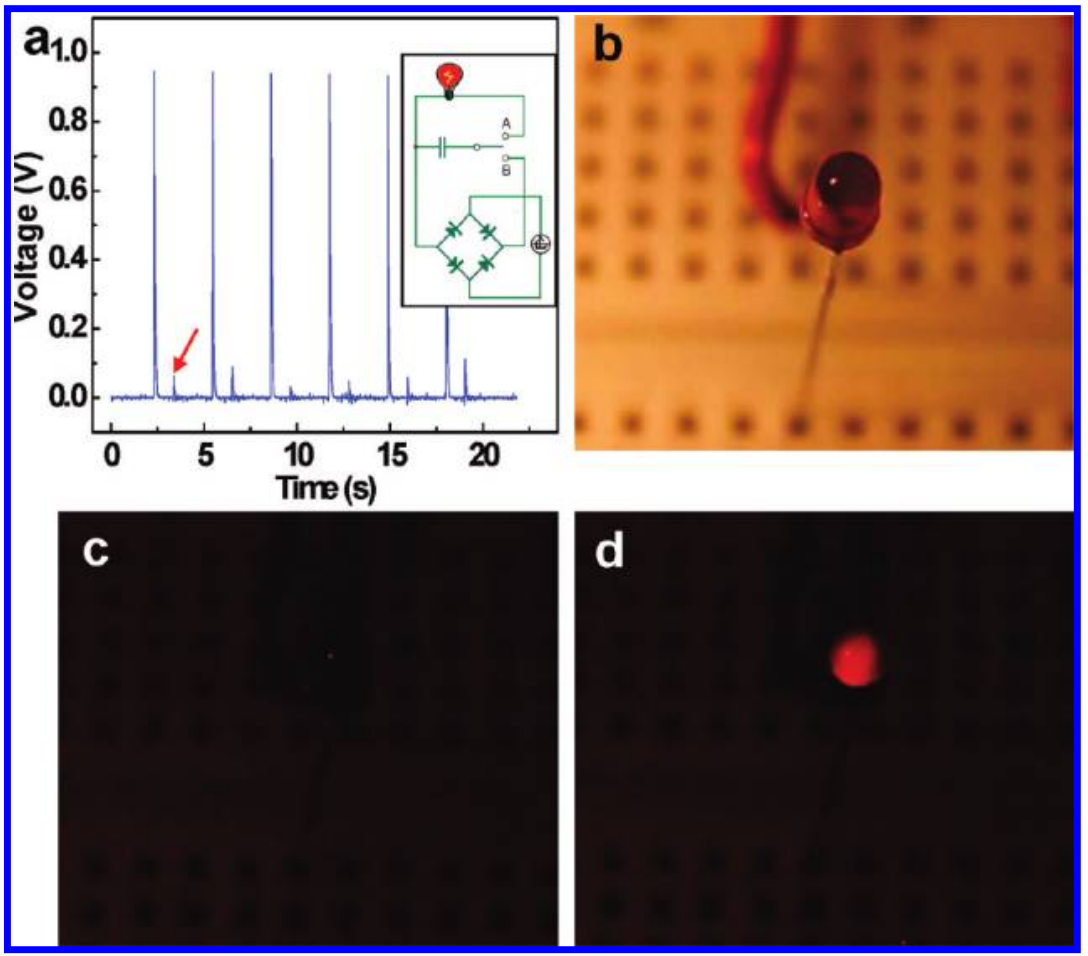

FIGURE 4. Application of the electric energy generated by the HONG to drive a commercial light emitting diode. (a) The electric output measured after a full wave rectifying bridge. Signals of negative signs are reversed, as pointed by the arrowhead. Inset: Schematic of the chargingdischarging circuit for storing and releasing the energy generated by the HONG, respectively. (b) Image of a commercial LED, which is incorporated into the circuit. (c) Image of the LED in dim background before it was lit up. (d) Image of the LED in dim background at the moment when it was lit up by the energy generated from the HONG.

switch is at position A for energy storage achieved by charging capacitors. Upon charging completion, the switch is switched to position B for energy releasing to power a functional device, such as a light emitting diode.
It is the key for a successful and effect energy storage to take full advantage of the alternating output. As a result, an integrated full wave rectifying bridge (Transys Electronics Limited, DI 102) was connected between a HONG and 
capacitors (Vishay Sprague, Type 430P, $2 \mu \mathrm{F} \pm 10 \%$ ). The output of the HONG measured after the bridge exhibits only positive signals (Figure 4a). Full wave rectification achieved by the bridge ensures energy storage at an enhanced efficiency, although the rectified signal (as pointed by an arrowhead in Figure 4a) has appreciably reduced magnitude due to the reverse current leakage of the diodes in the bridge; this reducing effect is rather notable at small output current. To facilitate the charging process, the output frequency of the HONG was tuned up to $3 \mathrm{~Hz}$ by reducing the periodicity of the mechanical deformation. Ten capacitors were connected in parallel such that they were simultaneously charged, and the voltage across a single capacitor finally reached 0.37 $\mathrm{V}$.

Upon finishing charging, the capacitors were reconfigured from parallel connection to series connection, leading to a total voltage source of $3.7 \mathrm{~V}$. The stored electricity was used to drive a commercial red LED (Figure 4b, Avago Technologies US Inc., HLMP-1700), which has an emission spectrum centered at $635 \mathrm{~nm}$. The turn-on voltage and forward-biased resistance are $1.7 \mathrm{~V}$ and $450 \Omega$, respectively. The discharging process was triggered, leading to a maximum discharging current of $4.5 \mathrm{~mA}$ and the LED was lit up. The emitted light lasted $0.1-0.2 \mathrm{~s}$ and was clearly captured in dim background (Figure 4c,d, video in Supporting Information). During the whole charging-discharging process, no other power sources were involved. The entire circuit is essentially a complete self-powered system, which consists of three components: an energy harvester (the HONG), storage units (capacitors), and a functional device (the LED).

An effective energy generation efficiency is defined as the ratio between the energy stored by the capacitors and the strain energy input to all of the active NWs, and it takes into account the performance of the electronic components in the circuit. The total electrical energy stored by the capacitor can be calculated as $W_{\text {stored }}=C U^{2} n / 2=1.37 \mu \mathrm{J}$, where $C$ is the capacitance of a single capacitor, $U$ is voltage across the capacitor, and $n$ is the number of capacitors. Since the dominant strain in the ZnO NWs is tensile strain, with shear strain safely neglected, the total strain energy can be estimated as $W_{\text {strain }}=\pi D^{2} L_{0} E \varepsilon^{2} f t n_{0} / 8=30 \mu \mathrm{J}$, where $D$ is the diameter of the NW (200 nm), $L_{0}$ is its original length (10 $\mu \mathrm{m})$, which is fixed by the electrode spacing, $E$ is the Young's modulus ( $30 \mathrm{GPa}), \varepsilon$ is the strain of NWs $(0.1 \%), f$ is the frequency of deformation ( $3 \mathrm{~Hz}), t$ is the total charging time (7200 s), and $n_{0}$ is the number of integrated NWs (300 000) ${ }^{13}$ Therefore, the effective energy generation efficiency is estimated to be $\sim 4.6 \%$. This value is naturally lower than the energy conversion efficiency of a single nano/ microwire $(\sim 7 \%)$, which is defined as the ratio between the generated electric energy $\left(W_{\text {generated }}=\int V I \mathrm{~d} t\right.$, where $V$ is the voltage, and $I$ is the current) and the mechanical input strain energy. ${ }^{13}$ This is mainly attributed to the energy dissipation on rectifying bridge and capacitors, as elaborated in the Supporting Information.
In summary, we have successfully fabricated high-output flexible nanogenerators using a sweeping-printing method. We managed to transfer vertically grown $\mathrm{ZnO} N W s$ to a flexible substrate and achieved horizontally aligned NW arrays that have crystallographic alignment, based on which an innovatively designed HONG was fabricated. The electrical output of the HONG reached a peak voltage of $2.03 \mathrm{~V}$ and current of $107 \mathrm{nA}$ with a peak power density of $\sim 11$ $\mathrm{mW} / \mathrm{cm}^{3}$, which is $12-22$ times of that from PZT-based cantilever energy harvester. An effective energy generation efficiency of $4.6 \%$ was demonstrated. The electric energy generated by the HONG was effectively stored by capacitors and used to light up a commercial LED. Furthermore, by optimizing the density of the NWs on the substrate and with the use of multilayer integration, a peak output power density of $\sim 0.44 \mathrm{~mW} / \mathrm{cm}^{2}$ and volume density of $1.1 \mathrm{~W} / \mathrm{cm}^{3}$ are redicted. This is a key step that is likely to bring nanogenerator based self-powering technology into people's daily life with potential applications in mobile electronics, health monitoring, environmental inspection, cargo shipping tracking system, infrastructure monitoring, and even defense technology.

Acknowledgment. Research supported by NSF (DMS 0706436, CMMI 0403671, ENG/CMMI 112024), DARPA (Army/AMCOM/REDSTONE AR), BES DOE (DE-FG0207ER46394), and DARPA/ARO W911NF-08-1-0249. The authors thank Cheng Li and Benjamin Hansen for their help on thin film deposition and electric circuit design, respectively.

Supporting Information Available. Experimental setup, additional figures, and video. This material is available free of charge via the Internet at http://pubs.acs.org.

\section{REFERENCES AND NOTES}

(1) Wang, Z. L. Sci. Am. 2007, 298, 82-87.

(2) Tian, B. Z; ; et al. Nature 2007, 449, 885-890

(3) Pan, C. F.; et al. Adv. Mater. 2008, 20, 1644-1648.

(4) Hausler, E.; et al. Ferroelectronics 1984, 60, 277-282.

(5) Platt, S. R.; et al. IEEE-ASME T. Mech. 2005, 10, 455-461.

(6) Round, S.; Wright, P. K.; Rabaey, J. Comput. Commun. 2003, 26, $1131-1144$.

(7) Shen, D. N.; et al. Sens. Actuators, A 2009, 154, 103-108

(8) Wang, Z. L.; Song, J. H. Science 2006, 312, 242-246.

(9) Wang, X. D.; Song, J. H.; Liu, J.; Wang, Z. L. Science 2007, 316, $102-105$

(10) Chang, C.; Tran, V. H.; Wang, J.; Fuh, Y.; Lin, L. Nano Lett. 2010 , 10, 726-731

(11) Qin, Y.; Wang, X. D.; Wang, Z. L. Nature 2008, 451, 809-813.

(12) Qi, Y; et al. Nano Lett. 2010, 10, 524-528.

(13) Yang, R.; Qin, Y.; Dai, L.; Wang, Z. L. Nat. Nanotechnol. 2009, 4, 34-39.

(14) Xu, S.; Qin, Y.; Xu, C.; Wei, Y. G.; Yang, R.; Wang, Z. L. Nat. Nanotechnol. 2010, 5, 367-273.

(15) Choi, D.; et al. Adv. Mater. 2010, 22, 2187-2192.

(16) Pan, Z. W.; Dai, Z. R.; Wang, Z. L. Science 2001, 291, 1947-1949

(17) Kuo, T.; et al. Chem. Mater. 2007, 19, 5143-5147.

(18) Polyakov, A. Y.; et al. Appl. Phvs. Lett. 2003, 83, 1575-1577.

(19) Yang, R.; Qin, Y.; Li, C.; Dai, L.; Wang, Z. L. Appl. Phys. Lett. 2009, 94, No. 022905.

(20) Agrawal, R.; Peng, B.; Espinosa, H. D. Nano Lett. 2009, 9, 4177 4183. 